\title{
Adjunctive Use of Calcipotriene/Betamethasone Dipropionate Foam in a Real-World Setting Curtails the Cost of Biologics Without Reducing Efficacy in Psoriasis
}

Jerry Bagel (1] · Elise Nelson · James Zapata · Alexa Hetzel

Received: August 25, 2020 / Published online: October 13, 2020

(C) The Author(s) 2020

\section{ABSTRACT}

Introduction: Although the efficacy of monotherapy with biologics for psoriasis is well established, many patients fail to achieve complete plaque clearance from their initial biologic treatment alone. Adjunctive treatment with topical calcipotriene plus betamethasone dipropionate (Cal/BD) foam may offer substantial clinical benefit and potential cost savings.

Methods: We conducted a 16-week, open-label, single-arm study of adjunctive therapy with Cal/BD foam in subjects who had been treated with etanercept or adalimumab for $\geq 24$ weeks but had not obtained a satisfactory treatment response. Assessments included affected body surface area (BSA), Physician's Global Assessment (PGA) of disease severity, BSA $\times$ PGA, National Psoriasis Foundation (NPF) Treat to Target status, and likelihood of the physician to switch biologics. In parallel, a cost analysis was performed to compare the cost of switching to a different biologic versus adding Cal/BD foam to the original biologic.

Results: Four weeks of daily adjunctive treatment with $\mathrm{Cal} / \mathrm{BD}$ foam led to notable reductions in BSA, PGA, and BSA $\times$ PGA relative to baseline. Additionally, by week $4,>75 \%$ of subjects achieved NPF Treat to Target status, and the likelihood of the investigator to switch biologics decreased from $90.0 \%$ at baseline to $7.1 \%$. The improved efficacy was maintained throughout the additional 12 weeks of maintenance Cal/BD foam application. The pharmacoeconomic evaluation demonstrated that adjuvant use of Cal/BD foam led to cost savings compared with switching biologic treatments.

Limitations: Due to the nature of the open-label study lacking a vehicle-treated control, no statistical comparison can be made.

Conclusions: The results of this study demonstrate that the addition of Cal/BD foam to plaque psoriasis patients who still have significant disease activity despite being on stable biologic therapy improves treatment outcomes to the point where switching to a more expensive biologic therapy is a less suitable treatment option.

Keywords: Adalimumab; Adjunctive therapy; Biologic therapy; Calcipotriene/betamethasone dipropionate (Cal/BD); Cost analysis; Etanercept; Observational study; Plaque psoriasis; Topical therapy
J. Bagel $(\bowtie) \cdot$ E. Nelson · J. Zapata · A. Hetzel

Psoriasis Treatment Center of New Jersey, East

Windsor, NJ, USA

e-mail: Dreamacres1@aol.com 


\section{Key Summary Points}

\section{Why carry out this study?}

While biologic monotherapy has had great success in treating moderate-to-severe psoriasis, there are many patients who are unable to achieve Treat to Target goals and optimal quality-of-life improvements

Such patients are faced with the decision of switching to a newer, and often more costly, biologic agent or maintaining their current regimen with an adjunctive therapy

What did the study ask?/What was the hypothesis of the study?

This open-label study examined the clinical benefits of adjunctive treatment with calcipotriene/dipropionate (Cal/BD) topical foam in patients who were unable to achieve satisfactory clearance of their psoriasis following $\geq 24$ weeks of biologic monotherapy and also assessed the potential cost savings of this adjunctive treatment approach versus switching to a different biologic agent

\section{What were the study outcomes/conclusions?}

Four weeks of daily adjunctive treatment with Cal/BD foam in a real-world setting resulted in improvements in all disease severity assessments, with $>75 \%$ of treated patients achieving Treat to Target status, and was found to yield substantial cost savings compared with switching to a new biologic treatment in a projected cost comparison with other commonly prescribed biologics

\section{What has been learned from the study?}

The results of this study demonstrate that the addition of Cal/BD foam to plaque psoriasis patients who still have significant disease activity despite being on stable biologic therapy improves treatment outcomes to the point where switching to a more expensive biologic therapy is a less suitable option

\section{DIGITAL FEATURES}

This article is published with digital features to facilitate understanding of the article. You can access the digital features on the article's associated Figshare page. To view digital features for this article go to https://doi.org/10.6084/m9. figshare.12961742.

\section{INTRODUCTION}

Psoriasis is a chronic immunologic disease that primarily affects the skin and joints [1-3]. It is estimated to have a prevalence of $2 \%$ to $4 \%$ both in the US [2,3] and worldwide [4], making it one of the most prevalent inflammatory immunologic diseases.

Plaque psoriasis is the most common form of the disease, affecting $80 \%$ to $90 \%$ of patients and manifesting as sharply demarcated, scaling, and erythematous lesions that vary in shape and size $[2,5,6]$. The lesions may be painful and are often severely pruritic [6]. In addition to the cutaneous symptoms, psoriasis has a strong psychologic impact that can substantially decrease a patient's quality of life (QoL) and daily functioning $[2,6,7]$. The impact of psoriasis on health-related QoL measures may be similar to that of other major medical diseases, including cancer and heart disease [7]. Additionally, psoriasis and its associated comorbidities, such as cardiometabolic, rheumatologic, and psychiatric conditions, can incur significant health care costs $[4,8]$. It is estimated that psoriasis costs 11 billion dollars annually in the US [9], thereby imposing a strong economic burden on the affected individuals and posing a significant public health problem.

As there is no cure for psoriasis, treatments aim to control clinical symptoms and improve the patient's QoL $[1,10]$. Individual treatment plans are guided by disease severity, which is determined by accounting for the extent of cutaneous symptoms as well as any disease-related impairments in daily functioning and QoL $[11,12]$. Commonly utilized assessments of psoriasis severity include the percent of affected body surface area (BSA) and the Physician's Global Assessment (PGA) of plaque severity [6]. 
BSA can account for the extent of cutaneous symptoms, such that mild, moderate, and severe psoriasis are defined by $<3 \%, 3 \%$ to $10 \%$, and $>10 \%$ BSA involvement [12], with the patient's full handprint estimating $1 \%$ of the total BSA [13]. The PGA provides an overall estimate of disease severity based on the appearance of the plaques $[6,12]$, and it is generally scored on a 5-point scale that includes categories for severe $(\mathrm{PGA}=4)$, moderate $(\mathrm{PGA}=3)$, mild $(\mathrm{PGA}=2)$, almost clear $(\mathrm{PGA}=$ $1)$, and clear $(P G A=0)$. In addition, the BSA $\times$ PGA composite score is a relatively novel tool that encompasses features of disease severity that are addressed by each of the BSA and PGA tools individually [14]. In general, it has become standard practice to incorporate multiple measures of psoriasis severity in clinical trials [15] to more fully capture the physical attributes of the disease. Treatment-based decisions should also include QoL considerations; the Dermatology Life Quality Index (DLQI) is one such tool that has widespread use and has been shown to increase the informative value of clinical research in psoriasis [13].

An appropriate treatment can be prescribed once the clinician has determined the disease severity and the extent to which it is impacting the patient's life. Topical medications or phototherapy are often sufficient for controlling symptoms in patients with milder disease $[10,16]$. However, treatments for patients with moderate-to-severe psoriasis usually require more extensive therapeutic interventions, such as systemic oral agents or biologic agents $[4,10,16]$. Biologic agents in particular have taken the forefront in treating moderate-tosevere psoriasis because of their favorable riskbenefit ratio [10, 16]. Regardless of disease severity and the corresponding regimen that is prescribed, a "Treat to Target" strategy in which the therapeutic intervention achieves $\mathrm{a} \leq 1 \%$ BSA involvement within 3 months of initiation is advocated by the National Psoriasis Foundation (NPF) [10, 17]. An additional treatment goal is to improve the patient's QoL to a level where it is either unaffected (DLQI score $0-1$ ) or affected to only a small degree (DLQI score 2-5) [13]. It is important to consider both of these treatment goals, as the extent of the affected area relates to the disease's impact on QoL; specifically, a decrease in BSA involvement of $\geq 90 \%$ is associated with greater improvements in QoL [18].

In practice, while biologic monotherapy has had great success in treating moderate-to-severe psoriasis, there are many patients who are unable to achieve NPF Treat to Target goals and optimal QoL improvements [4]. A recent study found that only one in four patients achieve complete skin clearance after 6 months of realworld treatment with biologics [19]. Additionally, more than one-half of all psoriasis patients are dissatisfied with their treatments [9], and the low patient satisfaction rate is particularly evident among those with moderate-to-severe psoriasis [11].

There are two general treatment options that can be utilized to improve disease outcomes and QoL measures in psoriasis patients with inadequate response to biologic monotherapy: switch to an alternative biologic agent or maintain the current biologic regimen but with an adjunctive therapy. Switching to a newer biologic agent entirely may help patients obtain NPF Treat to Target goals, but as the efficacy of second- and third-generation products has increased, so has the cost. Thus, switching to a more expensive biologic agent may not be a feasible option for many patients, given that the inability to obtain adequate insurance coverage is among the top reasons for discontinuing biologic therapy altogether [9]. Additionally, the physician may wish to retain the same biologic that has already been shown to be safe in their patient. A combination approach is often adopted to circumvent the limitations that are associated with switching or increasing the dose of the biologic while improving the overall treatment response [4]. Patients who lack a complete response to biologic therapy can benefit from the addition of other treatment modalities, including the addition of a systemic therapy or phototherapy, but long-term safety data on these treatment combinations are limited. Adjunctive use of topical agents may also be beneficial, especially for patients for whom ongoing therapy is recommended and an adequate response cannot be obtained with the current biologic alone [10]. 
Topical vitamin D analogs, corticosteroids, and these drugs in combinations are cornerstones of treatment for psoriasis patients $[10,12,20,21]$. Calcipotriene and betamethasone dipropionate (Cal/BD) foam is a fixed combination of calcipotriene $(50 \mathrm{mcg} / \mathrm{g})$, a vitamin $\mathrm{D}$ analog, and betamethasone dipropionate $(0.64 \mathrm{mg} / \mathrm{g})$, a corticosteroid $[20,22]$. The efficacy and safety of Cal/BD foam have previously been demonstrated in three Phase II/III clinical trials of plaque psoriasis of all severities $[20,23-25]$, and it is FDA-approved for oncedaily application for up to 4 weeks in the treatment of plaque psoriasis in patients $\geq 12$ years of age [22]. Notably, a recent study demonstrated that adjunctive therapy with $\mathrm{Cal} / \mathrm{BD}$ foam in patients with inadequate response to biologics resulted in improved disease outcomes, with the majority of patients achieving the NPF Treat to Target goal [26]. Adjunctive use of other Cal/BD formulations has also been shown to augment the initial response to biologics within the first 4 weeks of treatment initiation [27]. Furthermore, an ointment formulation of Cal/BD was found to be a safe and effective adjunct that helped maintain the long-term efficacy of biologic therapy after the regimen for the biologic was decreased to the long-term maintenance dosage [28]. Though adjunctive use of Cal/BD has demonstrated an added clinical benefit in these studies, patients' acceptance of the vehicle used in topical therapy can contribute to adherence to their treatment plan and ultimately impact treatment outcomes [12, 29]. Foam formulations are easy to apply and are preferred by patients over creams, ointments, and gels $[12,29]$. Together, these data suggest that adjunctive use of $\mathrm{Cal} / \mathrm{BD}$ foam may therefore be a valuable treatment option for patients who do not achieve sufficient clearance of psoriasis symptoms with biologic monotherapy.

An added benefit of adjunctive therapy with $\mathrm{Cal} / \mathrm{BD}$ foam versus switching the biologic is the potential cost savings for the patient and their insurance provider. The present study examined the efficacy and safety of $\mathrm{Cal} / \mathrm{BD}$ foam as adjunctive therapy to biologic treatment for moderate-to-severe psoriasis and also assessed the potential cost savings of this adjunctive treatment approach versus switching to a different biologic agent.

\section{METHODS}

\section{Study Design}

This was a single-center observational study of 30 subjects to assess 4 weeks of adjunctive therapy of Cal/BD foam once daily, followed by every-other-day Cal/BD foam for an additional 12 weeks, in subjects who had been receiving etanercept or adalimumab for at least 24 weeks but still had an affected BSA of $2 \%$ to $10 \%$ and a PGA score $\geq 2$ (Fig. 1). Cal/BD foam was applied daily for the initial 4 weeks of the study, consistent with its approved dosing [22]. Additional studies (e.g., the PSO-LONG study, NCT02899962) are currently under way to determine the long-term safety and efficacy of intermittent maintenance application of $\mathrm{Cal} / \mathrm{BD}$ foam for up to 1 year. However, without the availability of more long-term data involving intermittent application of Cal/BD foam, the dosage of $\mathrm{Cal} / \mathrm{BD}$ foam was reduced to every other day during weeks 4 to 16 to minimize potential risks that may be associated with longterm topical steroid use. Maintenance biologic therapy with either etanercept or adalimumab was continued throughout the 16-week study period (Fig. 1). Subjects attended scheduled visits at baseline (week 0) and weeks 4, 8, 12, and 16 . The screening period typically took place $0-30$ days prior to the baseline study visit unless a washout period was not required, in which case screening and baseline could have occurred on the same day.

Eligible subjects included male and nonpregnant female adults $\geq 18$ years of age with moderate-to-severe chronic plaque psoriasis who were treated with stable biologic therapy for $\geq 24$ weeks. Subjects were excluded if they had received an investigational drug, oral systemic medication, other topical therapies, or phototherapy within 2 to 4 weeks (dependent on the type of therapy) prior to study onset. The use of steroid-free topical emollients was permitted throughout the study, and appropriate interventions (e.g., prescribed medications) 


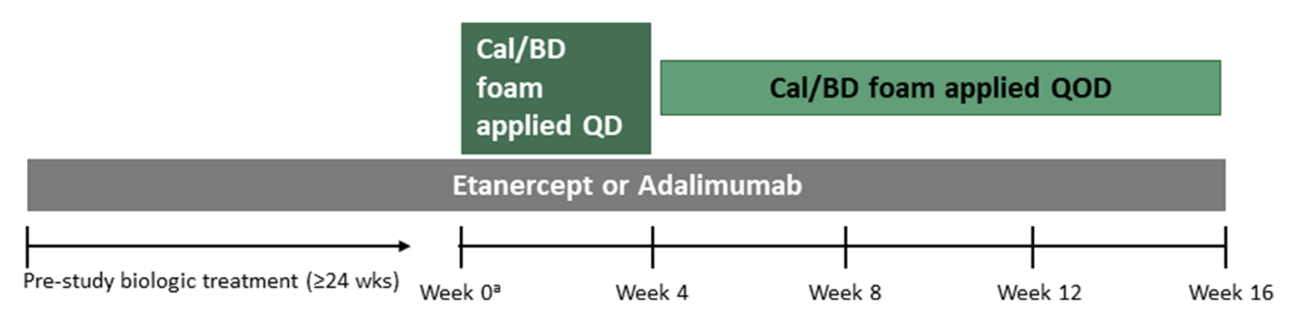

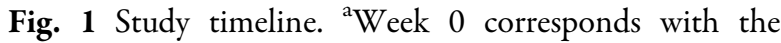
baseline of the open-label adjuvant Cal/BD study. Subject screening occurred after a minimum of 24 weeks of treatment with either etanercept or adalimumab, but prior

were performed as deemed necessary by the investigator to treat concomitant illnesses. Informed consent was obtained from each subject prior to participation in the study. Reasons for withdrawal from the study could include withdrawal by the subject, loss to follow-up, noncompliance with the study protocol, or other relevant reasons.

Written Institutional Review Board approval of the study protocol was obtained prior to study initiation. This study was conducted in accordance with the principles of the Declaration of Helsinki and with Good Clinical Practice guidelines.

\section{Planned Outcomes}

Safety and efficacy assessments were performed at all study visits. Disease severity outcome measures included mean BSA, mean PGA, and composite $\mathrm{BSA} \times$ PGA scores. Additional efficacy assessments included the percent of subjects that the investigator reported being likely to switch to an alternative biologic therapy and the percent of subjects achieving the NPF Treat to Target goal of BSA $\leq 1 \%$ within 3 months of starting treatment. While the Psoriasis Area and Severity Index (PASI) is commonly used to assess drug efficacy in clinical trials, it is rarely utilized in clinical practice and is known to have less sensitivity in patients with lower BSA involvement [21]. Thus, the PASI was not included as an assessment in the present study because it was carried out in a real-world setting in a population that had less extensive BSA involvement (see Table 1) due to the ongoing biologic therapy. The minimum required to the baseline study visit. Cal/BD calcipotriene/betamethasone dipropionate, $Q D$ every day, $Q O D$ every other day

24-week period of stable biologic monotherapy prior to initiating adjunctive $\mathrm{Cal} / \mathrm{BD}$ foam exceeded the 3-month treatment period that the NPF uses to guide treatment outcomes. This helped to ensure that subjects truly failed to achieve sufficient clinical outcomes prior to study entry rather than just having slower than usual onset of efficacy of etanercept or adalimumab.

Patient-reported outcomes included the Dermatology Life Quality Index (DLQI) and the Itch Numeric Rating Scale (NRS). The DLQI is a ten-item questionnaire that generates a score of 0-30; higher scores correlate with higher levels of impairment to QoL, with a score $\geq 10$ indicating that the subject's life is severely affected by their skin disease. The Itch NRS is an analog scale ranging from $0-10$ in which 0 indicates "no itch" and 10 indicates "the worst itch imaginable." Questionnaires were completed prior to medical procedures and clinical evaluations.

For safety evaluations, physical examinations were performed, including measurement of vital signs. Tolerability was evaluated by the investigator by assessing localized skin reactions for itching, dryness, burning/stinging, skin atrophy, striae, telangiectasia, and folliculitis. Adverse events (AEs) were captured as observations from the investigator or events reported by the subject, regardless of causality.

\section{Statistical Analysis}

All analyses were performed by the investigator. No comparator statistical testing was performed for the open-label efficacy data, and all data 
Table 1 Baseline demographics and disease characteristics

\begin{tabular}{|c|c|}
\hline Baseline characteristic & $\begin{array}{l}\text { Cal/BD foam } \\
(N=30)\end{array}$ \\
\hline \multicolumn{2}{|l|}{ Age (years) } \\
\hline Mean (SD) & $57.0(13.3)$ \\
\hline Median (range) & $58.5(27-87)$ \\
\hline \multicolumn{2}{|l|}{ Sex, $n(\%)$} \\
\hline Male & $18(60.0)$ \\
\hline Female & $12(40.0)$ \\
\hline \multicolumn{2}{|l|}{ Race, $n(\%)$} \\
\hline White & $21(70.0)$ \\
\hline Black & $3(10.0)$ \\
\hline Other & $6(20.0)$ \\
\hline \multicolumn{2}{|l|}{ Ethnicity, $n(\%)$} \\
\hline Hispanic or Latino & $6(20.0)$ \\
\hline Not Hispanic or Latino & $24(80.0)$ \\
\hline $\begin{array}{l}\text { PSO duration (years), mean } \\
\text { (SD) }\end{array}$ & $25.4(14.9)$ \\
\hline \multicolumn{2}{|l|}{ Biologic, $n(\%)$} \\
\hline Adalimumab & $27.0(90.0)$ \\
\hline Etanercept & $3(10.0)$ \\
\hline \multicolumn{2}{|l|}{ PGA, $n(\%)$} \\
\hline PGA $=2$ (mild) & $10(33.3)$ \\
\hline PGA $=3$ (moderate $)$ & $20(66.7)$ \\
\hline \multicolumn{2}{|l|}{ BSA \% } \\
\hline Mean (SD) & $4.8(2.3)$ \\
\hline Median (range) & $4(2-10)$ \\
\hline NPF treat to target status, $n(\%)$ & $0(0.0 \%)$ \\
\hline
\end{tabular}

Baseline refers to the initial study visit (week 0) that occurred after subjects had been treated with etanercept or adalimumab for $\geq 24$ weeks

$B S A$ body surface area, Cal/BD calcipotriene/betamethasone dipropionate, NPF National Psoriasis Foundation, $P G A$ Physician's Global Assessment, PSO psoriasis, SD standard deviation were reported for observed cases only, with no imputations for missing data. Safety assessments were based on descriptive statistics; no inferential tests were performed.

\section{Pharmacoeconomic Evaluation of Biologic Therapies}

For the cost projection, we compared the cumulative cost of adding Cal/BD foam to etanercept or adalimumab versus switching to a new biologic agent. The former was obtained by adding the cost of 16 weeks of maintenance dosing with the biologic to the incremental increase associated with adding 4 weeks of once-daily dosing of Cal/BD foam followed by 12 weeks of once-every-other-day dosing. To calculate the cost of switching to a new biologic, we used average wholesale prices for the most commonly prescribed biologics multiplied by the dosing recommended for the induction and maintenance phases of up to 16 weeks of treatment. Prices were obtained from the 2019 RED BOOK ${ }^{\circledR}$ wholesale acquisition cost database [30] and the 2020 average wholesale price specialty pharmacy reports.

\section{RESULTS}

\section{Baseline Demographics and Disease Characteristics}

A total of 30 subjects were included in the study, with a little more than half of the subjects being male $(60.0 \%)$ and the majority being white $(70.0 \%)$ (Table 1$)$. The subjects had had a diagnosis of chronic plaque psoriasis for an average of 25.4 years and had been undergoing treatment with either etanercept $(10.0 \%)$ or adalimumab (90.0\%) for a minimum of 24 weeks prior to the study baseline. The subjects had significant disease activity at baseline despite being on stable biologic therapy: $66.7 \%$ of subjects had moderate psoriasis severity as assessed by PGA scores, with a mean affected BSA of $4.8 \%$; $0 \%$ of subjects had achieved NPF Treat to Target status after $\geq 24$ weeks of monotherapy with etanercept or adalimumab. 


\section{Disease Severity Outcome Measures}

Once-daily application of Cal/BD foam for 4 weeks resulted in notable improvements in all assessed disease severity outcomes. The mean affected BSA decreased from $4.8 \%$ to $1.4 \%$ (Fig. 2a), and the mean PGA decreased from 2.67 to 1.04 (Fig. 2b), demonstrating that 4 weeks of adjunctive therapy with Cal/BD foam improved psoriasis severity on average from moderate to mild in the pooled population. Similarly, there was a pronounced improvement in the mean composite BSA $\times$ PGA scores (Fig. 2c), which decreased from 12.9 to 1.5 after the first 4 weeks of daily Cal/BD foam application. After the switch from once-daily Cal/BD application to a maintenance schedule of once every other day, there were modest increases in affected BSA, PGA scores, and composite BSA $\times$ PGA scores (Fig. 2). However, overall, the improvements in all three disease severity outcome measures remained relatively constant and never approached baseline levels (Fig. 2). By the end of the study at week 16, BSA and PGA remained reduced by $>50 \%$ of the baseline scores, and the composite BSA $\times$ PGA score was reduced by $>80 \%$ of baseline, clearly demonstrating the beneficial effect of adjunctive $\mathrm{Cal} / \mathrm{BD}$ foam.

At baseline, none of the subjects had achieved the NPF Treat to Target goal with either etanercept or adalimumab monotherapy (Fig. 3). Adding once-daily application of

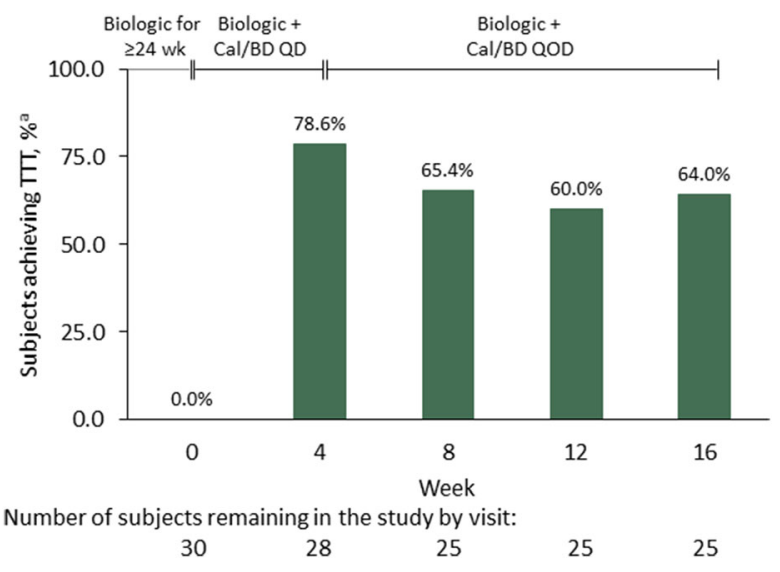

Fig. 3 Percentage of subjects achieving NPF Treat to Target status, by study visit. ${ }^{2}$ Percentages are calculated from observed cases at each study visit. Cal/BD calcipotriene/betamethasone dipropionate, $Q D$ every day, $Q O D$ every other day, TTT NPF Treat to Target

Cal/BD foam to the subjects' ongoing biologic treatment regimens for 4 weeks resulted in $>75 \%$ of the study population's achieving NPF Treat to Target status (Fig. 3). Most of the subjects were able to maintain their NPF Treat to Target status throughout the study duration even after decreasing Cal/BD application to once every other day. From baseline to week 16, the percent of the population that achieved NPF Treat to Target status increased from 0 to $64.0 \%$ (Fig. 3).

At the beginning of the study, the study investigators reported being likely to switch biologics because of failure to achieve the NPF

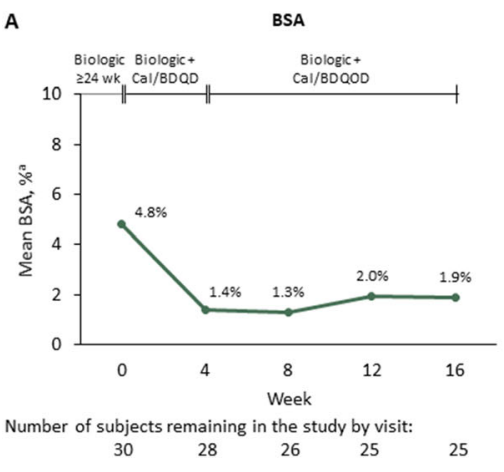

Fig. 2 Mean BSA (a), PGA (b), and composite BSA $\times$ PGA scores (c) plotted over the course of the 16-week study. ${ }^{a}$ Means are calculated from observed cases at each study visit. $B S A$ body surface area, Cal/BD
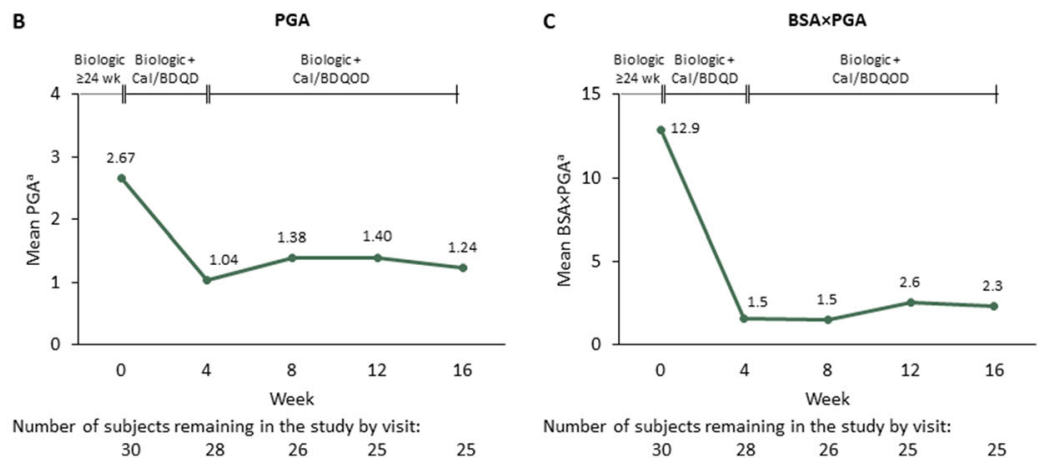

calcipotriene/betamethasone dipropionate, $P G A$ Physician's Global Assessment, $Q D$ every day, $Q O D$ every other day 
Treat to Target goal for $90.0 \%$ of the subjects (Fig. 4). Adjunctive Cal/BD foam greatly reduced the likelihood of switching biologics: by week 4 , the percentage of subjects for whom the study investigators reported being likely to switch to an alternative biologic was only $7.1 \%$. The added benefit of Cal/BD foam to ongoing treatment with etanercept or adalimumab remained relatively constant throughout the study duration, with investigators being likely to switch biologics for $12.0 \%$ of subjects by week 16 (Fig. 4).

\section{Patient-Reported Outcome Measures}

At baseline, $26.7 \%$ of subjects reported a DLQI score of 0 or 1 (Fig. 5a), indicating that QoL was still negatively impacted by psoriasis in most subjects despite having been treated with etanercept or adalimumab for at least 24 weeks. After 4 weeks of adjunctive therapy with Cal/BD foam, the percentage of subjects with a DLQI score of 0 or 1 nearly doubled, suggesting substantial improvements in QoL. The percentage of subjects reporting a DLQI score of 0 or 1 continued to increase throughout the study duration, with $64.0 \%$ of subjects reporting this score by week 16 (Fig. 5a).

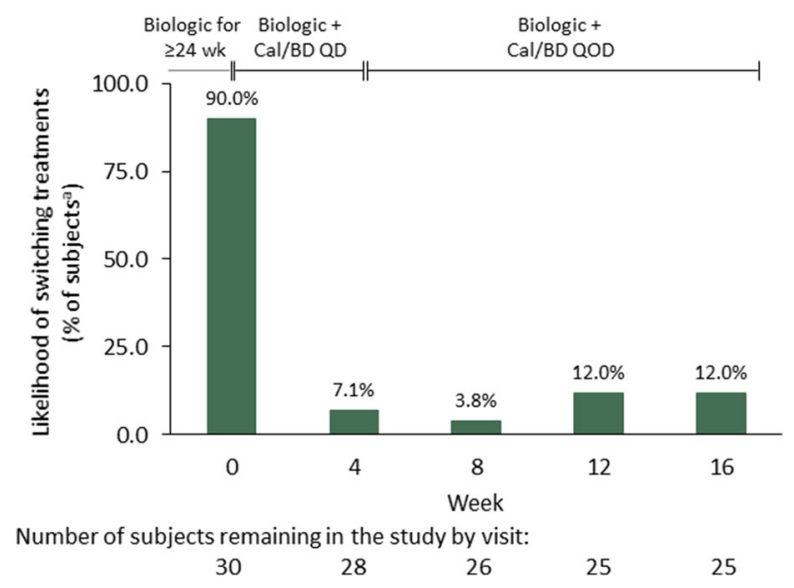

Fig. 4 Percentage of subjects for whom the physician reported being likely to switch biologics, by study visit. ${ }^{a}$ Percentages are calculated from observed cases at each study visit. Cal/BD calcipotriene/betamethasone dipropionate, $Q D$ every day, $Q O D$ every other day
Improvements were also observed in patientreported itch symptoms following the addition of Cal/BD foam. The mean Itch NRS score was 4.5 at baseline (Fig. 5b), indicating moderate levels of itch-associated discomfort at the beginning of the study. Mean itch ratings decreased to 1.4 and 1.5 by weeks 4 and 16, respectively. Thus, 4 weeks of daily $\mathrm{Cal} / \mathrm{BD}$ foam application was associated with improvements in itch ratings that were maintained up to week 16 even after the switch to every-otherday application (Fig. 5b).

\section{Safety Outcomes}

Overall, few AEs were reported (Table 2). The most frequently reported $\mathrm{AE}$ was the common cold $(6.7 \%)$. There was one case of contact dermatitis that was considered to be treatment related; all other AEs were considered unrelated to study treatment. All AEs were graded as mild in severity, except for one case of basal cell carcinoma (moderate severity).

The application of Cal/BD foam once daily and every other day was well tolerated, as determined by local skin reaction assessments (Fig. 6). The percentage of subjects in whom itching, burning/stinging, and dryness was reported as none or mild increased from baseline to week 4 and remained increased up to week 16.

\section{Pharmacoeconomics of Switching Biologic Therapies Versus Adding Cal/BD Foam}

Table 3 summarizes the costs associated with the most commonly prescribed biologics that were prescribed and sent through a large mail order specialty pharmacy (Bioplus Specialty Pharmacy), as well as the costs for the most recently prescribed biologics, as derived from average wholesale prices from May 2019 through May 2020. These 16-week cost estimates were based on induction dosing according to manufacturer recommendations plus subsequent maintenance dosing through 16 weeks for all agents except etanercept and adalimumab; costs for etanercept and 


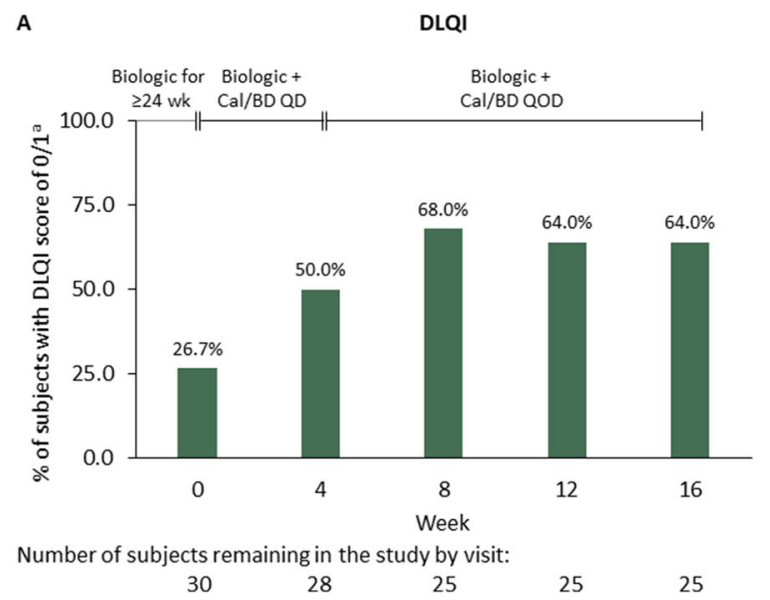

Fig. 5 Patient-reported outcomes, by study visit. ${ }^{\text {a Means }}$ and ${ }^{b}$ percentages are calculated from observed cases at each study visit. Cal/BD calcipotriene/betamethasone

Table 2 Summary of adverse events

\begin{tabular}{llll}
\hline Adverse event & $N(\%)^{\mathbf{a}}$ & Severity & $\begin{array}{c}\text { Treatment- } \\
\text { relatedness }\end{array}$ \\
\hline Common cold & $2(6.7)$ & Mild & Not related \\
Cellulitis & $1(3.3)$ & Mild & Not related \\
Sinus infection & $1(3.3)$ & Mild & Not related \\
Contact & $1(3.3)$ & Mild & Related \\
dermatitis & $1(3.3)$ & Mild & Not related \\
Cough & $1(3.3)$ & Mild & Not related \\
Nightmares & $1(3.3)$ & Mild & Not related \\
Bleeding on left \\
elbow
\end{tabular}

a Percentages are calculated based on total number of enrolled subjects $(N=30)$

adalimumab were based solely on maintenance dosing for 16 weeks, as those were the two agents that subjects were already receiving

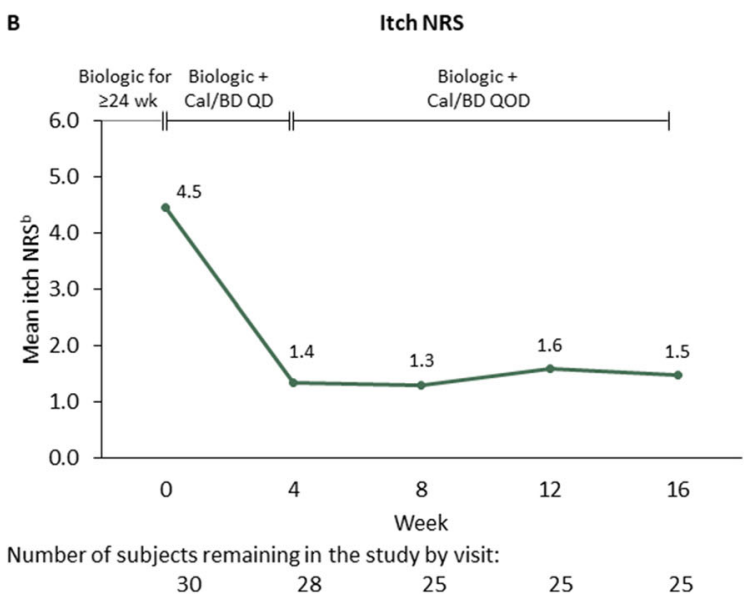

dipropionate, DLQI Dermatology Life Quality Index, $N R S$ numeric rating scale, $Q D$ every day, $Q O D$ every other day

for $\geq 24$ weeks. The estimated 16 -week cost of maintenance treatment with etanercept or adalimumab plus adjunctive $\mathrm{Cal} / \mathrm{BD}$ foam was $\$ 25,046$ and $\$ 26,062$, respectively. By comparison, switching to any of the other five most commonly prescribed biologics was associated with higher cost. For example, switching to ustekinumab at the lowest approved dose (45 mg) was estimated to cost $\$ 36,177$, more than $\$ 10,000$ greater over 16 weeks than adding $\mathrm{Cal} / \mathrm{BD}$ foam. The most expensive of the other commonly prescribed biologics was risankizumab, at an estimated 16-week cost of $\$ 96,270$, followed by secukinumab at $\$ 92,720$, ixekizumab at $\$ 53,550$, and guselkumab at $\$ 47,608$.

\section{DISCUSSION}

This open-label study examined the clinical benefits of adjunctive use of $\mathrm{Cal} / \mathrm{BD}$ foam in a real-world setting in subjects with significant psoriasis disease activity despite being on stable biologic therapy for 24 weeks or more. The level of their persistent disease activity warranted either adding therapy or switching to a different biologic agent. At the beginning of the study, investigators reported being likely to switch biologic agents for $90.0 \%$ of subjects. Among the subjects, $73.3 \%$ indicated that their QoL was adversely affected, highlighting the 

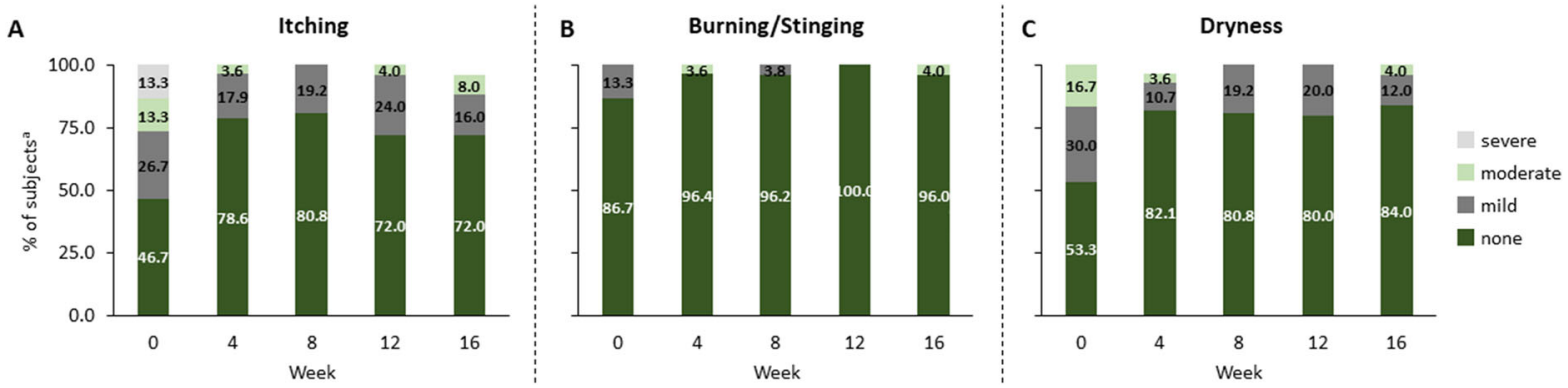

Fig. 6 Percentage of subjects with localized skin reactions showing symptoms of itching (a), burning/stinging (b), and

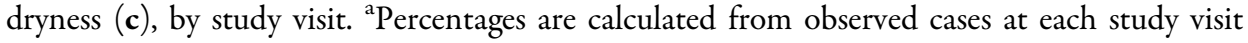

need to modify treatment strategies in this population to improve their clinical outcomes. The results of this single-arm, observational study demonstrated that the addition of topical $\mathrm{Cal} / \mathrm{BD}$ foam to the existing biologic treatment was a safe and effective alternative to switching to a different biologic and additionally yielded substantial cost savings.

The subjects in this study were unable to achieve satisfactory clearance of their psoriasis with either etanercept or adalimumab for $\geq 24$ weeks. Combination therapy with $\mathrm{Cal} / \mathrm{BD}$ foam resulted in improvements in all assessed disease severity measures after the initial 4-week induction period with daily application. There were notable reductions in affected BSA, PGA scores, and composite BSA $\times$ PGA scores within just 4 weeks of adjunctive therapy, and subjects were able to maintain these improvements for an additional 12 weeks of combination therapy using Cal/BD foam every other day. Importantly, these improved clinical outcomes were associated with high rates of achievement of NPF Treat to Target goals. While no subjects achieved NPF Treat to Target status with etanercept or adalimumab alone, by week 4 of combination therapy, $78.6 \%$ of subjects had achieved $\leq 1 \%$ BSA status, and $64.0 \%$ were able to maintain this treatment goal for up to 16 weeks. The patientreported outcomes provided additional support for the benefit of adding Cal/BD foam to their ongoing biologic treatments, as the proportion of subjects with a DLQI score of 0 or 1 increased from $26.7 \%$ to $50.0 \%$ by week 4 , and it reached $64.0 \%$ by the end of the study. Taking into account the high rates of subjects who achieved
NPF Treat to Target status and those whose QoL improved, the investigators were highly likely to continue biologic therapy with etanercept or adalimumab in combination with Cal/BD foam instead of switching to a different biologic.

Previous studies have investigated the use of topical therapy with biologics for psoriasis and have demonstrated that the combination regimen provides added clinical benefits to biologic monotherapy [26-28]. The results of this study provide additional support for the use of combination therapy with topicals and demonstrated that the addition of Cal/BD foam provided better disease control than either etanercept or adalimumab alone.

The potential cost savings of adding Cal/BD foam versus switching to a new biologic were evident from a projected cost comparison with other commonly prescribed biologic agents. Combining Cal/BD foam with either etanercept or adalimumab would result in greater cost savings than switching to any of the commonly prescribed, commercially available biologics. Depending on which alternative biologic agent was chosen, continuing with the initial biologic and adding Cal/BD foam would result in a cost savings ranging between $\$ 11,131$ and $\$ 71,224$ over the course of a 16-week treatment period. Thus, adding Cal/BD foam to ongoing biologic therapy with etanercept or adalimumab would help the majority of patients achieve NPF Treat to Target goals at a much lower cost than switching to a new biologic. Alternatively, switching to a biosimilar might also provide a more affordable option to switching biologics. However, none of the FDA-approved biosimilars for etanercept or adalimumab are currently 
Table 3 Sixteen-week cost estimate for switching biologics versus adding Cal/BD foam

\begin{tabular}{|c|c|c|c|c|}
\hline Biologic & Total cost ${ }^{\mathrm{a}}$ & Dose & Cost $/$ dose & No. of doses ${ }^{b}$ \\
\hline Etanercept (Enbrel $®)$ & $\$ 22,224.00$ & $50 \mathrm{mg}$ & $\$ 1389.00$ & 16 \\
\hline Etanercept $+\mathrm{Cal} / \mathrm{BD}$ foam $\left(\text { Enstilar }{ }^{\circledR}\right)^{\mathrm{c}}$ & $\$ 25,046.00$ & & & \\
\hline Adalimumab (Humira ${ }^{\circledR}$ ) & $\$ 23,240.00$ & $40 \mathrm{mg}$ & $\$ 2905.00$ & 8 \\
\hline Adalimumab + Cal/BD foam & $\$ 26,062.00$ & & & \\
\hline \multicolumn{5}{|l|}{ Ustekinumab (Stelara $\left.{ }^{\circledR}\right)$} \\
\hline $45 \mathrm{mg}$ dosage & $\$ 36,177.00$ & $45 \mathrm{mg}$ & $\$ 12,059.00$ & 3 \\
\hline $90 \mathrm{mg}$ dosage & $\$ 72,354.00$ & $90 \mathrm{mg}$ & $\$ 24,118.00$ & 3 \\
\hline Guselkumab (Tremfya ${ }^{\circledR}$ ) & $\$ 47,608.00$ & $100 \mathrm{mg}$ & $\$ 11,902.00$ & 4 \\
\hline Ixekizumab (Taltz $\left.{ }^{\circledR}\right)$ & $\$ 53,550.00$ & $80 \mathrm{mg}$ & $\$ 5950.00$ & 9 \\
\hline Secukinumab (Cosentyx $\left.{ }^{\circledR}\right)$ & $\$ 92,720.00$ & $300 \mathrm{mg}$ & $\$ 11,590.00$ & 8 \\
\hline Risankizumab (Skyrizi $\left.{ }^{\circledR}\right)$ & $\$ 96,270.00$ & $150 \mathrm{mg}$ & $\$ 32,090.00$ & 3 \\
\hline
\end{tabular}

${ }^{a}$ Cost estimates for the most recently prescribed biologics are based on average wholesale prices from May 2019 to May 2020. Estimates for the most frequently prescribed biologic agents that have been prescribed and sent through a large mailorder specialty pharmacy (Bioplus Specialty Pharmacy) are given for that same period. Biologics are listed in order of the least expensive to the most expensive option among these commonly prescribed agents. The estimates reflect the cost of induction and subsequent maintenance for all biologic agents except etanercept and adalimumab, which would only require the continued maintenance cost

${ }^{\mathrm{b}}$ Includes the number of doses required for induction and maintenance during 16 weeks of treatment for all biologics except etanercept and adalimumab, which only require maintenance dosing

c Cal/BD foam: $\$ 1411 / 60 \mathrm{~g}$ canister; average, 2 cans/patient in a 16-week period

$\mathrm{Cal} / \mathrm{BD}$ calcipotriene/betamethasone dipropionate

commercially available, and it is not clear that any such agents would provide added efficacy beyond that of etanercept or adalimumab. Therefore, the augmented clinical outcomes that are associated with adjunctive Cal/BD foam, together with its cost savings, make it a desirable treatment option for psoriasis patients undergoing biologic therapy who still have unmet needs.

There were few limitations to the study. The cost inputs of the analysis represent wholesale acquisition costs (WACs) and not the net price paid by health plans or patients after discounts. Discounts from WACs can include manufacturer/health plan negotiated contracts, manufacturer rebates to health plans, and manufacturer-sponsored patient assistance programs. However, discounted rates for products have wide variation between health plans, which makes applying standard discount adjustments across multiple products in pharmacoeconomic evaluations less acceptable [31]. Thus, in the absence of known discount rates, the pharmacoeconomic component of this analysis utilized WACs.

Other limitations relate to the nature of the study design. This was a single-center study involving a relatively small patient population, and therefore the results may not be representative of all patient types. Additionally, in this open-label study, all subjects received $\mathrm{Cal} / \mathrm{BD}$ foam. Thus, no statistical inference can be made between the efficacy of adjunctive use of Cal/BD foam with etanercept or adalimumab and the continued use of etanercept or adalimumab alone, nor between the adjunctive regimen and switching to a different biologic monotherapy. However, it is unlikely that continuing 
monotherapy with etanercept or adalimumab or switching to an alternative biologic agent would have achieved appreciably better results than those observed with Cal/BD foam, given the large percentage of patients who were able to achieve the NPF Treat to Target goal with adjunctive Cal/BD treatment. Nonetheless, these results demonstrate that subjects were able to achieve noteworthy clinical improvements with adjunctive Cal/BD foam and etanercept/adalimumab in a real-world setting.

\section{CONCLUSIONS}

This real-world study demonstrated that Cal/BD foam was an effective, safe, and well-tolerated adjunctive topical therapy in subjects with moderate-to-severe psoriasis who still had significant psoriasis disease activity despite having been on stable biologic therapy for $\geq 24$ weeks. Topical Cal/BD foam may be an ideal adjunctive treatment to maintenance therapy with etanercept or adalimumab that is more affordable than switching to other commercially available biologic agents.

\section{ACKNOWLEDGEMENTS}

Funding. Funding of this study and the Journal's Rapid Service Fee was provided by LEO Pharma.

Authorship. All named authors meet the International Committee of Medical Journal Editors (ICMJE) criteria for authorship for this article, take responsibility for the integrity of the work as a whole, and have given their approval for this version to be published.

Authorship Contributions. All authors contributed to the study design, data collection, and analysis. All authors have contributed to the manuscript preparation and have approved of the manuscript.

Medical Writing and Editorial Assistance. $P$-value communications provided medical writing, editing, and publication assistance and was funded by LEO Pharma.

Disclosures. Jerry Bagel has received research funds payable to Psoriasis Treatment Center from AbbVie, Amgen, Arcutis Biotherapeutics, Boehringer Ingelheim, Bristol Myers Squibb, Celgene Corporation, Corrona LLC, Dermavant Sciences, LTD, Dermira/UCB, Eli Lilly and Company, Glenmark Pharmaceuticals Ltd, Janssen Biotech, Kadmon Corporation, LEO Pharma, Lycera Corp, Menlo Therapeutics, Novartis, Pfizer, Regeneron Pharmaceuticals, Sun Pharma, Taro Pharmaceutical Industries Ltd, and Valeant Pharmaceuticals; consultant fees from AbbVie, Amgen, Celgene Corporation, Eli Lilly and Company, Janssen Biotech, LEO Pharma, Novartis, Sun Pharmaceutical Industries Ltd, and Valeant Pharmaceuticals; and fees for speaking from AbbVie, Celgene Corporation, Eli Lilly, Janssen Biotech, LEO Pharma, and Novartis. Elise Nelson has received consultant fees from Eli Lilly and Company. James Zapata has no financial interests to declare. Alexa Hetzel has received fees for speaking for Sun Pharmaceutical Industries Ltd. and AbbVie.

Compliance with Ethics Guidelines. Written Institutional Review Board approval of the study protocol was obtained prior to study initiation. This study was conducted in accordance with the principles of the Declaration of Helsinki and with Good Clinical Practice guidelines. Informed consent was obtained from each subject prior to participation in the study

Data Availability. The datasets generated during and/or analyzed during the current study are available from the corresponding author on reasonable request.

Open Access. This article is licensed under a Creative Commons Attribution-NonCommercial 4.0 International License, which permits any non-commercial use, sharing, adaptation, distribution and reproduction in any medium or format, as long as you give appropriate credit to the original author(s) and the source, provide a link to the Creative Commons licence, and indicate if changes were made. The images or 
other third party material in this article are included in the article's Creative Commons licence, unless indicated otherwise in a credit line to the material. If material is not included in the article's Creative Commons licence and your intended use is not permitted by statutory regulation or exceeds the permitted use, you will need to obtain permission directly from the copyright holder. To view a copy of this licence, visit http://creativecommons.org/licenses/by$\mathrm{nc} / 4.0 /$.

\section{REFERENCES}

1. Greaves MW, Weinstein GD. Treatment of psoriasis. N Engl J Med. 1995;332(9):581-8.

2. Langley RG, Krueger GG, Griffiths CE. Psoriasis: epidemiology, clinical features, and quality of life. Ann Rheum Dis. 2005;64 Suppl 2:ii18-23 (discussion ii24-25).

3. Takeshita J, Gelfand JM, Li P, et al. Psoriasis in the us medicare population: prevalence, treatment, and factors associated with biologic use. J Invest Dermatol. 2015;135(12):2955-63.

4. Armstrong AW, Bagel J, Van Voorhees AS, et al. Combining biologic therapies with other systemic treatments in psoriasis: evidence-based, best-practice recommendations from the medical board of the National Psoriasis Foundation. JAMA Dermatol. $2015 ; 151(4): 432-8$.

5. Griffiths CE, Christophers E, Barker JN, et al. A classification of psoriasis vulgaris according to phenotype. Br J Dermatol. 2007;156(2):258-62.

6. Menter A, Gottlieb A, Feldman SR, et al. Guidelines of care for the management of psoriasis and psoriatic arthritis: Section 1. Overview of psoriasis and guidelines of care for the treatment of psoriasis with biologics. J Am Acad Dermatol. 2008;58(5): 826-850.

7. Rapp SR, Feldman SR, Exum ML, et al. Psoriasis causes as much disability as other major medical diseases. J Am Acad Dermatol. 1999;41(3 Pt 1): 401-7.

8. Rachakonda TD, Schupp CW, Armstrong AW. Psoriasis prevalence among adults in the United States. J Am Acad Dermatol. 2014;70(3):512-6.

9. Armstrong $\mathrm{AW}$, Robertson $\mathrm{AD}, \mathrm{Wu} \mathrm{J}$, et al. Undertreatment, treatment trends, and treatment dissatisfaction among patients with psoriasis and psoriatic arthritis in the United States: findings from the National Psoriasis Foundation surveys, 2003-2011. JAMA Dermatol. 2013;149(10):1180-5.

10. Bagel J, Gold LS. Combining topical psoriasis treatment to enhance systemic and phototherapy: a review of the literature. J Drugs Dermatol. 2017;16(12):1209-22.

11. Korman NJ, Zhao Y, Lu J, et al. Psoriasis disease severity affects patient satisfaction with treatment. Dermatol Online J. 2015;21(7).

12. Stein Gold LF. Topical therapies for psoriasis: improving management strategies and patient adherence. Semin Cutan Med Surg. 2016;35(2 Suppl 2):S36-S44 (quiz S45).

13. Finlay AY. Current severe psoriasis and the rule of tens. Br J Dermatol. 2005;152(5):861-7.

14. Stein Gold L, Hansen JB, Patel D, et al. PGAxBSA composite versus PASI: comparison across disease severities and as therapeutic response measure for $\mathrm{Cal} / \mathrm{BD}$ foam in plaque psoriasis. J Am Acad Dermatol. 2020;83(1):131-8.

15. Robinson A, Kardos M, Kimball AB. Physician global assessment (PGA) and psoriasis area and severity index (PASI): why do both? A systematic analysis of randomized controlled trials of biologic agents for moderate to severe plaque psoriasis. J Am Acad Dermatol. 2012;66(3):369-75.

16. Menter A, Strober BE, Kaplan DH, et al. Joint AADNPF guidelines of care for the management and treatment of psoriasis with biologics. J Am Acad Dermatol. 2019;80(4):1029-72.

17. Strober BE, van der Walt JM, Armstrong AW, et al. Clinical goals and barriers to effective psoriasis care. Dermatol Ther (Heidelb). 2019;9(1):5-18.

18. Bruins FM, Bronckers I, Groenewoud HMM, et al. Association between quality of life and improvement in psoriasis severity and extent in pediatric patients. JAMA Dermatol. 2019;156(1):72-8.

19. Seneschal J, Lacour JP, Bewley A, et al. A multinational, prospective, observational study to estimate complete skin clearance in patients with moderateto-severe plaque PSOriasis treated with BIOlogics in a REAL world setting (PSO-BIO-REAL). J Eur Acad Dermatol Venereol. 2020.

20. Leonardi C, Bagel J, Yamauchi P, et al. Efficacy and safety of calcipotriene plus betamethasone dipropionate aerosol foam in patients with psoriasis vulgaris-a randomized phase III study (PSO-FAST). J Drugs Dermatol. 2015;14(12):1468-77. 
21. Menter A, Korman NJ, Elmets CA, et al. Guidelines of care for the management of psoriasis and psoriatic arthritis. Section 3. Guidelines of care for the management and treatment of psoriasis with topical therapies. J Am Acad Dermatol. 2009;60(4): 643-659.

22. Enstilar ${ }^{\circledR}$ (calcipotriene and betamethasone dipropionate) foam [package insert]. Madison, NJ: LEO Pharma Inc; 2019.

23. Lebwohl M, Tyring $S$, Bukhalo $M$, et al. Fixed combination aerosol foam calcipotriene $0.005 \%$ (Cal) plus betamethasone dipropionate $0.064 \%$ (BD) is more efficacious than Cal or BD aerosol foam alone for psoriasis vulgaris: a randomized, double-blind, multicenter, three-arm, phase 2 study. J Clin Aesthet Dermatol. 2016;9(2):34-41.

24. Paul C, Stein Gold L, Cambazard F, et al. Calcipotriol plus betamethasone dipropionate aerosol foam provides superior efficacy vs. gel in patients with psoriasis vulgaris: randomized, controlled PSO-ABLE study. J Eur Acad Dermatol Venereol. 2017;31(1):119-126.

25. Stein Gold L, Lebwohl M, Menter A, et al. Aerosol foam formulation of fixed combination calcipotriene plus betamethasone dipropionate is highly efficacious in patients with psoriasis vulgaris: pooled data from three randomized controlled studies. J Drugs Dermatol. 2016;15(8):951-7.

26. Bagel J, Zapata J, Nelson E. A prospective, open-label study evaluating adjunctive calcipotriene 0 . 005\%/betamethasone dipropionate $0.064 \%$ foam in psoriasis patients with inadequate response to biologic therapy. J Drugs Dermatol. 2018;17(8): 845-850.

27. Thaci D, Ortonne JP, Chimenti S, et al. A phase IIIb, multicentre, randomized, double-blind, vehiclecontrolled study of the efficacy and safety of adalimumab with and without calcipotriol/betamethasone topical treatment in patients with moderate to severe psoriasis: the BELIEVE study. Br J Dermatol. 2010;163(2):402-11.

28. Kircik LH. Topical calcipotriene $0.005 \%$ and betamethasone dipropionate $0.064 \%$ maintains efficacy of etanercept after step-down dose in patients with moderate-to-severe plaque psoriasis: results of an open label trial. J Drugs Dermatol. 2011;10(8):878-882.

29. Housman TS, Mellen BG, Rapp SR, et al. Patients with psoriasis prefer solution and foam vehicles: a quantitative assessment of vehicle preference. Cutis. 2002;70(6):327-32.

30. IBM Micromedex Red Book®. IBM.Com/products/ micromedex-red-book/pricing. Accessed 7 July 2020.

31. Mattingly TJ 2nd, Levy JF, Slejko JF, et al. Estimating drug costs: how do manufacturer net prices compare with other common us price references? Pharmacoeconomics. 2018;36(9):1093-9. 